\title{
Large Deflections Mechanical Analysis of a Suspended Single-Wall Carbon Nanotube under Thermoelectrical Loading
}

\author{
Assaf Ya'akobovitz, ${ }^{1}$ Slava Krylov, ${ }^{1}$ and Yael Hanein ${ }^{2}$ \\ ${ }^{1}$ School of Mechanical Engineering, Faculty of Engineering, Tel Aviv University, Ramat Aviv, Tel Aviv 69978, Israel \\ ${ }^{2}$ School of Electrical Engineering, Faculty of Engineering, Tel Aviv University, Ramat Aviv, Tel Aviv 69978, Israel
}

Correspondence should be addressed to Assaf Ya’akobovitz, assafyaa@eng.tau.ac.il

Received 14 July 2010; Revised 17 October 2010; Accepted 3 January 2011

Academic Editor: Sulin Zhang

Copyright (C) 2011 Assaf Ya'akobovitz et al. This is an open access article distributed under the Creative Commons Attribution License, which permits unrestricted use, distribution, and reproduction in any medium, provided the original work is properly cited.

\begin{abstract}
Following the recent progress in integrating single-wall carbon nanotubes (SWCNTs) into silicon-based micro-electromechanical systems (MEMS), new modeling tools are needed to predict their behavior under different loads, including thermal, electrical and mechanical. In the present study, the mechanical behavior of SWCNTs under thermoelectrical loading is analyzed using a large deflection geometrically nonlinear string model. The effect of the resistive heating was found to have a substantial influence on the SWCNTs behavior, including significant enhancement of the strain (up to the millistrains range) and buckling due to the thermal expansion. The effect of local buckling sites was also studied and was found to enhance the local strain. The theoretical and numerical results obtained in the present study demonstrate the importance of resistive heating in the analysis of SWCNTs and provide an additional insight into the unique mechanics of suspended SWCNTs.
\end{abstract}

\section{Introduction}

Integration of carbon nanotubes (CNTs), and especially single-wall carbon nanotubes (SWCNTs) into larger scale microelectromechanical (MEMS) silicon structures, is attractive due to the unique physical properties of CNTs. CNT technology appears to be beneficial in applications such as SWCNTs transistor [1], SWCNTs resonators [2], and SWCNTs-based biological, chemical [3], and mechanical sensors $[4,5]$.

The progress achieved in integrating methods of SWCNTs into silicon structures, including MEMS devices [4-6], set the groundwork for the creation of a new generation of MEMS sensors, which exploit the high sensitivity of SWCNTs along with the robustness and flexibility of the already well established MEMS technology. Several SWCNTMEMS sensors were reported in the literature. In these device physical stimuli (mechanical, electrical, magnetic, etc.) are applied to the MEMS device. The MEMS structures deform along with the SWCNT that serves as the sensing element. Due to the small dimensions the SWCNTs, even small deformations of a microstructure may generate a significant strain in an anchored nanotube, which results in a change in their electrical properties $[4,7]$. The sensors reported to date demonstrated high sensitivity and can be used as high end pressure sensors [5], displacement sensors [4], mass sensors $[8]$ and strain sensors $[9,10]$. Understanding the physics of suspended SWCNTs is, therefore, an important task towards the successful design of SWCNT-based devices.

Numerous studies were devoted to understanding SWCNT mechanics, including extensive experimental, as well as theoretical studies [11-14]. Several studies analyzed the effect of the mechanical deformation on the electrical $[4,15]$ or optical [16] properties of SWCNTs. A variety of approaches were used in modeling SWCNT mechanics, ranging from atomistic approaches, through mesoscale approaches, up to large scale continuum mechanics (CM) approaches. The most widely used atomistic approaches are molecular dynamics (MD) methods, which provide credible results [17]. However, MD methods are computationally expensive and thus limited to the modeling of very short SWCNTs (i.e., SWCNTs of several nanometers 


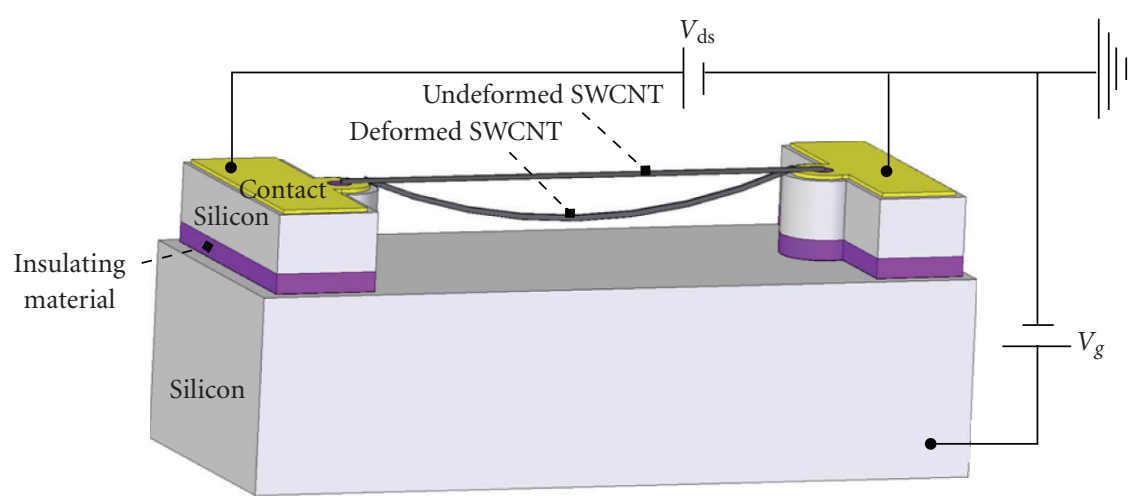

FIGURE 1: Schematics of the device under consideration. A SWCNT is suspended between two silicon anchors and is electrically insulated from the gate electrode.

in length [18]). Mesoscale approaches, such as coarse grained methods or the dissipative particle dynamics [19], enable the modeling of larger scale SWCNTs, but are still computationally expensive. The well established CM methods, on the other hand, are computationally cheaper and are not limited by the dimensions of the SWCNTs [11, 20]. In addition, $\mathrm{CM}$ approaches often result in relatively simple and compact models allowing analytical treatment and provides an insight into the behavior of CNTs. Although CM models do not take into account interatomic interactions within CNTs, several comparative studies have shown that $\mathrm{CM}$ results are in close agreement with results obtained from MD simulations [18] or experiments [11,21].

In spite of the vast variety of reported SWCNT modeling approaches, the effect of resistive heating on the mechanical deformation of a suspended SWCNT under electrostatic loading has yet to be analyzed. As we will show below, this effect has substantial influence on the SWCNT mechanics. Due to poor heat transfer mechanisms in suspended SWCNTs, the temperature of SWCNTs can rise by hundreds of degrees even with relatively small electrical currents [22].

In the present study, a large deflection, nonlinear sting model was used in order to simulate the behavior of suspended SWCNTs. Contrary to most previously reported studies in which SWCNTs were modeled as a beam, in the current study, SWCNTs were modeled as a string. A string model is justified by the high slenderness of realistic nanotube devices $[6,23,24]$. We begin with considering the nonlinear large deflection equation of equilibrium, followed by discussing the solution to this equation for a specific geometry. The effect of the resistive heating is then analyzed using the string model, followed by nonlinear finite element (FE) simulation to study of the influence of the local buckling sites on the strain arising in the SWCNTs. Finally, discussion and conclusions are presented.

\section{Formulation}

In the present study, the problem of a suspended SWCNT under resistive heating and electrostatic force was analyzed. The configuration of the device is shown schematically in Figure 1. The SWCNT is suspended on a silicon structure.
The undeformed configuration of the SWCNT can be either taut or slacked, as a function of the temperature and the prestress, as will be discussed below. Drain-source voltage, $V_{\mathrm{d}}$, is applied to the SWCNT and generates the resistive heating. In addition, gate voltage, $V_{g}$, is applied to the SWCNT by a silicon electrode which is electrically insulated from the SWCNT by a dielectric material, such as silicon dioxide or silicon nitride. The electrode is located at a relatively large distance from the SWCNT (several micrometers). The distance between the anchors (attachment points) is $L_{0}$. Such an experimental setup was reported by us in $[6,23,24]$.

Two main simplifying assumptions were made while developing the device model. First, we consider a SWCNT of high aspect ratio (long with respect to its cross-section). In this case, the SWCNT under consideration has low bending stiffness, the stiffness resulting from the axial tension of the SWCNT is dominant, and the device can be considered as a string. Second, we assume that the electrostatic force applied to the string is independent of its deflection. Strictly speaking, the electrostatic force applied to the SWCNT depends nonlinearly on the gap between the SWCNT and the electrode [25]. However, since the displacements of the SWCNT (in the order of hundreds of nanometers) are significantly smaller than the initial electrostatic gap (in the order of several micrometers or even tens of micrometers), we assume the electrostatic force to be displacement independent. In addition, since the gate voltage (in the order of tens of Volts) is significantly higher than the drain-source voltage (in the order of tenth of Volts), we consider the actuation voltage $V$ to be equal to the gate voltage $(V=$ $V_{g}$ ) and disregard the voltage difference along the SWCNT. Under these assumptions and taking into account the fact that the electrostatic force is perpendicular to the SWCNT axis, the deformed SWCNT has the form of a circular arc. Therefore, the governing equilibrium equation is

$$
N \kappa=q,
$$

where $N$ is the tension force, $q$ is the electrostatic force per unit length applied to the SWCNT, and $\kappa$ is the curvature of the deformed SWCNT.

The electrostatic force per unit length, $q$, was derived by calculating the derivative of the electrostatic coenergy 
$E=-C V^{2} / 2$, where $C$ is the capacitance per unit length of the straight tube and $V$ is the actuation voltage, with respect to the tube deflection. This capacitance was calculated as a capacitance of a wire of a diameter $d$, a length $L_{0}$ and an electrostatic gap $h$, located above an infinite plane [26]. The capacitance $C$ is given by

$$
C=\frac{2 \pi \varepsilon_{0}}{\ln \left(L_{0}^{2}\left(\sqrt{1+16 h^{2} / L_{0}^{2}}-1\right) / 2 d h\right)},
$$

where $\varepsilon_{0}$ is the dielectric permittivity of air.

The tension force, $N$, includes an initial pretension, $N_{0}$, which is related to the fabrication process, a tension resulting from the elongation (stretch) in the SWCNT during the deformation and a thermal compression force, namely,

$$
N=N_{0}+E A \epsilon-E A \alpha \Delta T .
$$

Here $E$ and $A=\pi d t$ are the Young's modulus and the SWCNT cross-section area, respectively (with $d$ and $t$ are the diameter and thickness of the SWCNT, resp.), $\alpha$ is the coefficient of thermal expansion (see [27]) and $\Delta T$ is the temperature difference with respect to a reference temperature, for example, ambient room temperature. We note that (3), which is the constitutive relation of the tube, incorporates an assumption that the strain within the SWCNT is small, the stress-strain relation is linear, and the nonlinearity is only of a geometric nature. The average strain induced by deformation is defined as follows:

$$
\epsilon=\frac{L}{L_{0}}-1
$$

where $L_{0}$ and $L$ are the undeformed and the deformed lengths of the tube, respectively. Note that $L_{0}$ corresponds to a state when the SWCNT has an initial tension of $N_{0}$ rather than being a stress-free SWCNT.

We note that due to the resistive heating, the string expands and the strain decreases below the initial pretension. For temperature differences higher than a certain value, $\Delta T_{\text {cr }}=N_{0} / \alpha E A$, the thermal strain and the initial tensile strain eliminate each other. Since the string cannot sustain a compressive force, a deflection (slackness) appears. In this case, the only tension force that governs the behavior of the SWCNT is the stretch (see (3)) due to the transversal electrostatic force. Therefore, we define two regimes of the SWCNT behavior. In the first, also referred to as the prebuckling regime, the temperature is below its critical value $\Delta T \leq \Delta T_{\mathrm{cr}}$, the SWCNT has initial length of $L_{0}$, and the constitutive relation and strain are governed by (3) and (4), respectively. In the second regime, also referred to as the postbuckling regime the axial tension is due to stretch, namely, the constitutive relation is

$$
N=E A \epsilon .
$$

Contrary to the prebuckling regime, where a temperature increase results in a decrease in the axial force, under the postbuckling regime, an increase in the temperature results in elongation of the SWCNT and a larger slackness.
We emphasize that for temperature differences lower than $\Delta T_{\text {cr }}$ (prebuckling regime) and in the absence of a transverse loading, the string is initially straight and the undeformed length $L_{0}$ is used in the calculation of the strain, in accordance with (4). However, for temperature differences higher than $\Delta T_{\text {cr }}$ (postbuckling regime), the SWCNT has an initial deflection (slackness). In this case, the strain is calculated in accordance with the expression

$$
\epsilon=\frac{L}{L_{T}}-1 .
$$

Here, $L_{T}$ is the SWCNT length for zero force and it is calculated as follows:

$$
L_{T}=L_{0}\left[1+\alpha\left(\Delta T-\Delta T_{\mathrm{cr}}\right)\right] .
$$

In this work the strain in the prebuckling regime is calculated using (4) while in the postbuckling regime it was calculated using (6). Note that the strain is commonly calculated with respect to the stress-free reference length. However, since in experimental characterization of such systems the measured electrical resistance is related to elongation, we calculate the strain with respect to the length corresponding to zero force.

The geometrical relation between the undeformed $\left(L_{0}\right)$, the deformed $(L)$ lengths of the stretched SWCNT and the curvature $(\kappa)$, for both aforementioned regimes, is expressed as follows:

$$
L_{0}=\frac{2}{\kappa} \sin \left(\frac{L \kappa}{2}\right) .
$$

Substituting the expression for the tension $N$ (the constitutive relation of (3) for prebuckling regime and (5) for the postbuckling regime) with strain (given by (4) for prebuckling regime and (6) for postbuckling regime) into the equilibrium (1) results in the following equilibrium equation for the case of prebuckling:

$$
\left(N_{0}+E A\left(\frac{L}{L_{0}}-1\right)-E A \alpha \Delta T\right) \kappa=q,
$$

whereas in the case of postbuckling, the equilibrium equation is

$$
E A\left(\frac{L}{L_{T}}-1\right) \kappa=q
$$

By expressing the term for the deformed length $L$ from (9) and (10) and substituting them into (8), we obtain the nonlinear algebraic equations relating the curvature of the string to the applied electrostatic force. Under prebuckling regime, the equation has the form of

$$
L_{0}=\frac{2}{\kappa} \sin \left(\frac{L_{0} \kappa}{2 E A}\left(\frac{q}{\kappa}-N_{0}\right)+\frac{L_{0} \kappa}{2}(1+\alpha \Delta T)\right),
$$

whereas under postbuckling regime the following equation is obtained

$$
L_{0}=\frac{2}{\kappa} \sin \left(\frac{L_{T} \kappa}{2}\left(\frac{q}{\kappa E A}+1\right)\right) .
$$




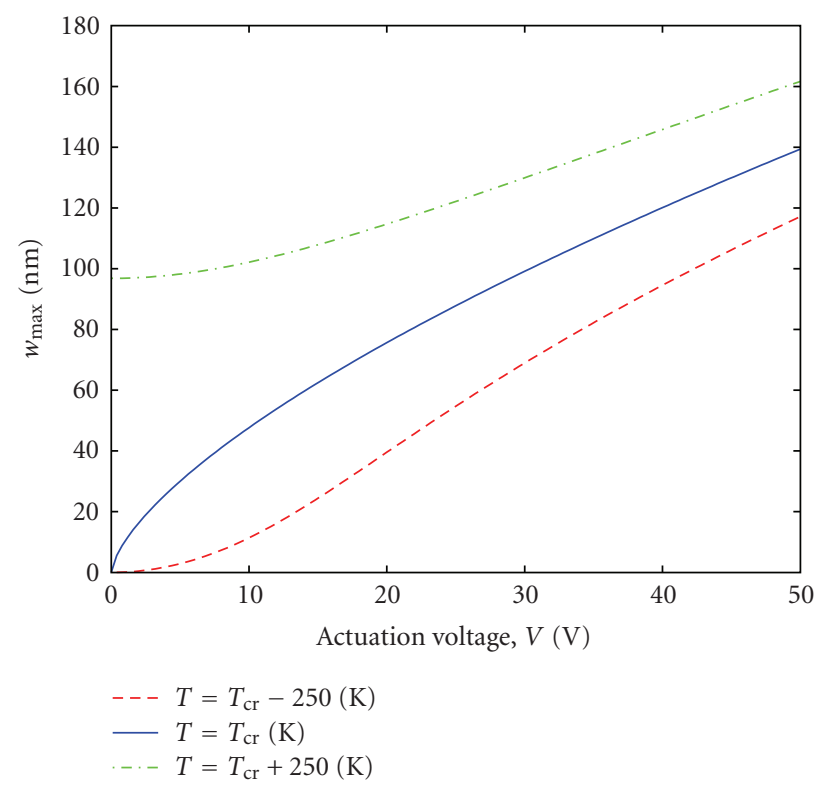

(a)

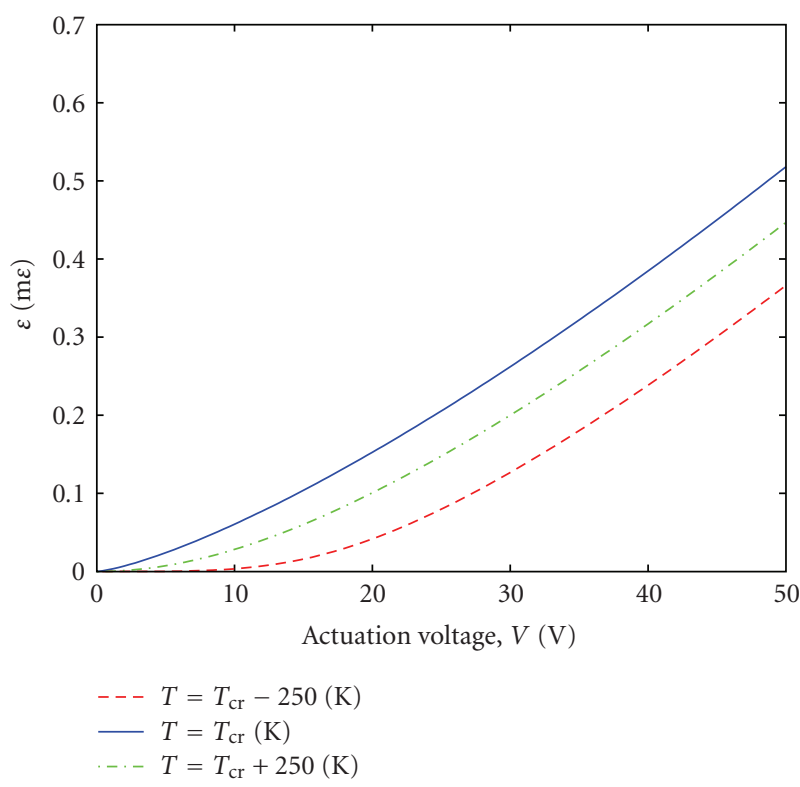

(b)

FIGURE 2: Forced response for a SWCNT with parameters listed in Tables 1 and 2 at different temperatures. (a) Maximum displacement. (b) Average axial strain.

TABLE 1: SWCNT geometrical parameters used in the calculations.

\begin{tabular}{lc}
\hline Parameter & Value \\
\hline Initial (unbuckled) length & $10 \mu \mathrm{m}$ \\
Thickness & $0.34 \mathrm{~nm}$ \\
Diameter & $4 \mathrm{~nm}$ \\
Electrostatic gap & $10 \mu \mathrm{m}$ \\
\hline
\end{tabular}

TABLE 2: Material properties used in the calculations.

\begin{tabular}{lc}
\hline Parameter & Value \\
\hline SWCNT Young's modulus, $E$ & $1 \mathrm{TPa}$ \\
SWCNT initial tension, $N_{0}$ (see [23]) & $1 \mathrm{nN}$ \\
Dielectric permittivity of the air, $\varepsilon_{0}$ & $8.854 \cdot 10^{-12} \mathrm{~F} / \mathrm{m}$ \\
Thermal expansion coefficient, $\alpha$ (see [27]) & $1 \cdot 10^{-6} \mathrm{~K}^{-1}$ \\
\hline
\end{tabular}

We note that the above mentioned model is nonlinear. This model takes into account large SWCNTs deflections, as well as a tension force, $N$, which depends on the SWCNT deflection. In general, the electrostatic load is also not linear. For simplicity, in the present study, it is considered to be deflection independent. We emphasize that several studies reported experiments which agree with the assumption presented above $[6,23,24]$.

\section{Model Results}

Equation (11) (for the case of $\Delta T \leq \Delta T_{\mathrm{cr}}$ ) or (12) (for the case of $\Delta T>\Delta T_{\text {cr }}$ ) is solved in terms of the curvature $\kappa$ using the Newton-Raphson-based solver incorporated into the MATLAB package. The geometrical dimensions as well

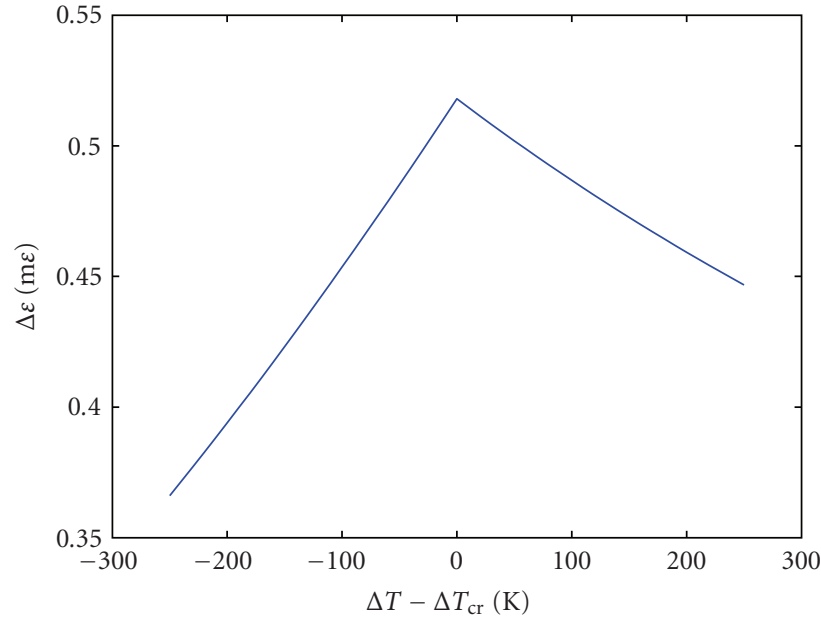

FIGURE 3: Average axial strain versus temperature with respect to the temperature difference $\Delta T-\Delta T_{\text {cr }}$ for a SWCNT with parameters listed in Tables 1 and 2. Constant actuation voltage of $50 \mathrm{~V}$ was applied to the SWCNT. Note that for negative $\Delta T-\Delta T_{\text {cr }}$ the strain was calculated using (4) while for positive $\Delta T-\Delta T_{\text {cr }}$ the strain was calculated using (6).

as the material constants used in the calculations are listed in Tables 1 and 2, respectively. After the deformed lengths were calculated using (8), deflections were calculated using geometrical relations and the average strain was calculated over the whole length of the SWCNT using (4) and (6).

The influence of the temperature on the SWCNT behavior was analyzed in Figures 2 and in 3. As mentioned, at the critical temperature, $\Delta T=\Delta T_{\mathrm{cr}}$, and at temperatures lower than the critical value, $\Delta T<\Delta T_{\text {cr }}$ (prebuckling 


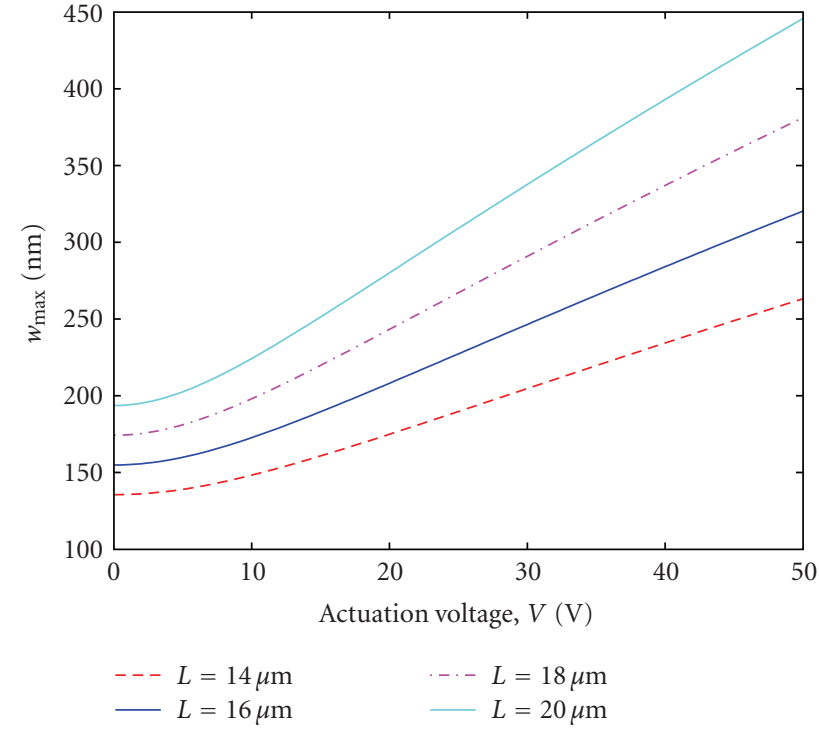

(a)

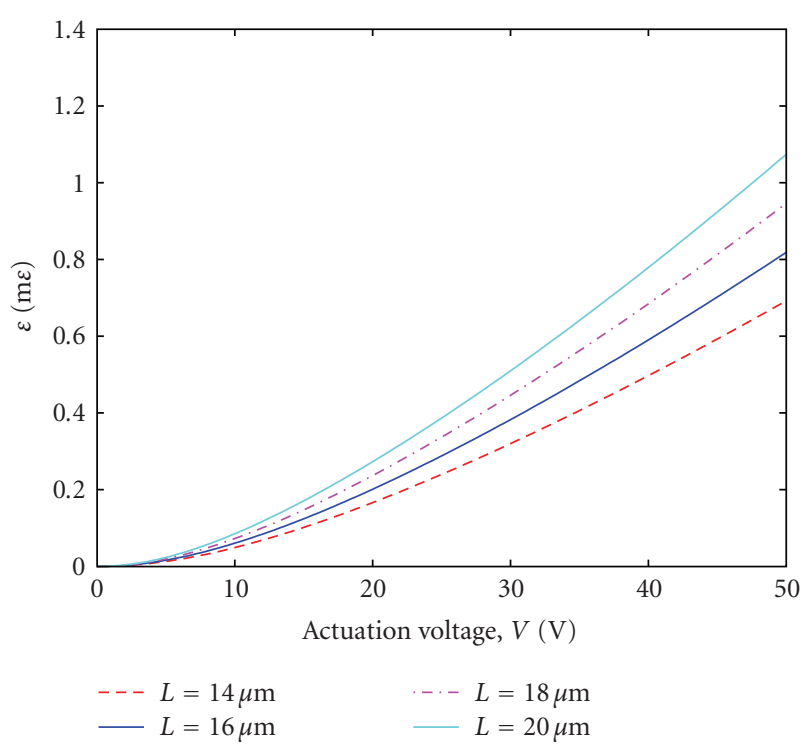

(b)

FIgURE 4: Forced response for SWCNT with parameters listed in Tables 1 and 2, $\Delta T_{K}=\Delta T_{\text {cr }}+250[K]$ and different lengths. (a) Maximum displacement. (b) Average axial strain.

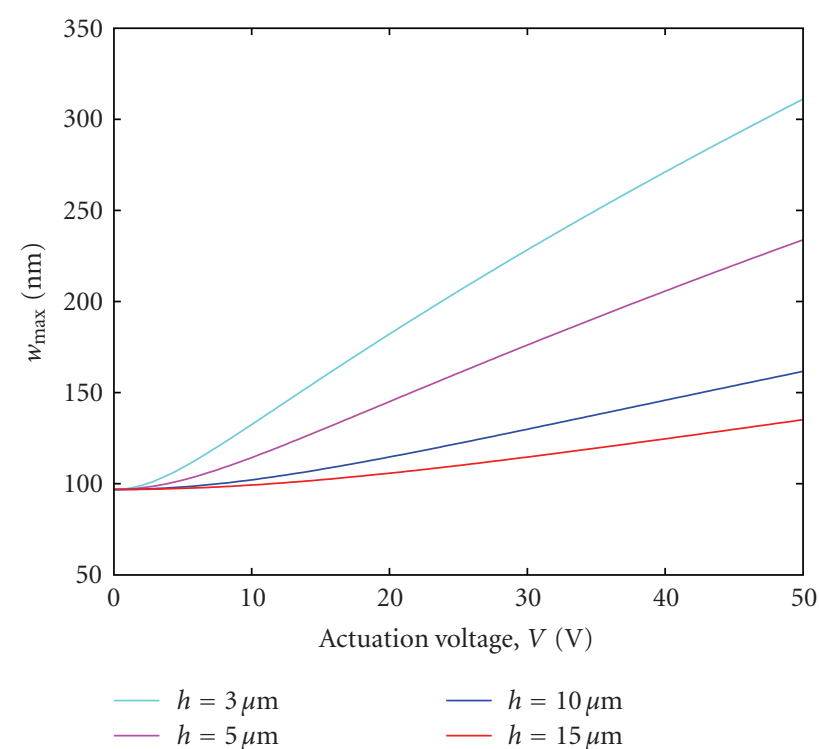

(a)

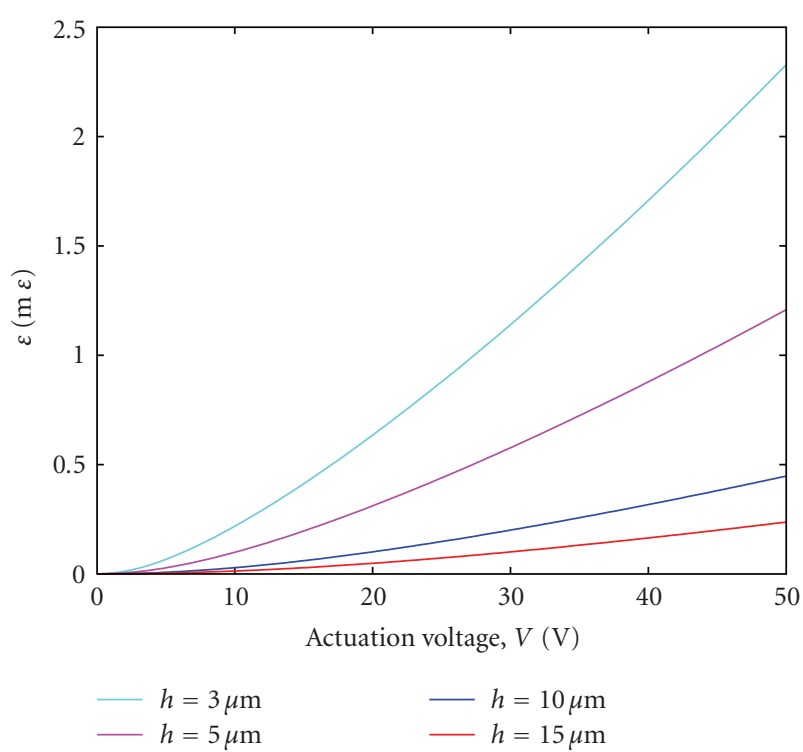

(b)

FIGURE 5: Forced response for SWCNT with parameters listed in Tables 1 and 2, $\Delta T_{K}=\Delta T_{\text {cr }}+250[K]$ and different electrostatic gaps. (a) Maximum displacement. (b) Average axial strain.

regime), the initial displacement of the SWCNT is zero. However, for temperatures higher than the critical temperature (postbuckling regime), a thermal buckling appears and the SWCNT has an initial nonzero displacement corresponding to infinitesimally small external load, Figure 2(a). Note that the maximum displacement (the displacements corresponding to the midpoint of the SWCNT) is in the order of hundreds of nanometers.

Figure 3 represents the strain of the SWCNT under a constant actuation voltage of $50 \mathrm{~V}$. It can be noticed that in the pre-buckled regime, the strain increases with temperature, namely, the temperature has a softening effect on the SWCNT. This can be explained by the fact that the compressive component of the axial force increases with the temperature, so that high temperatures are associated with SWCNT of lower tension. However, when thermal buckling takes place, an opposite trend of stiffening of the SWCNT was noticed. In this case, the SWCNT has initial slackness which results in higher stiffness since the axial force, which is horizontal before buckling takes place, has a component 


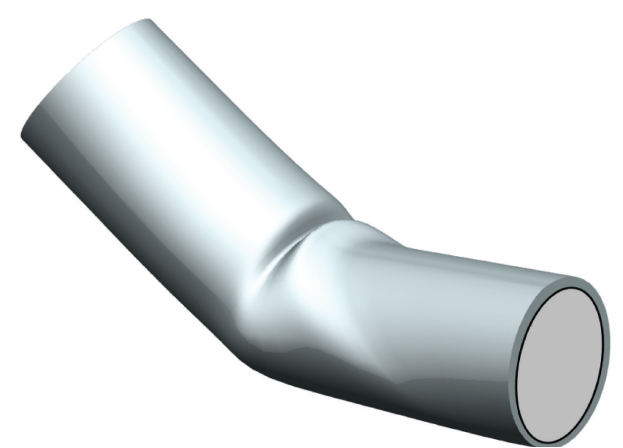

FIGURE 6: An illustrative image of a SWCNT buckling site.

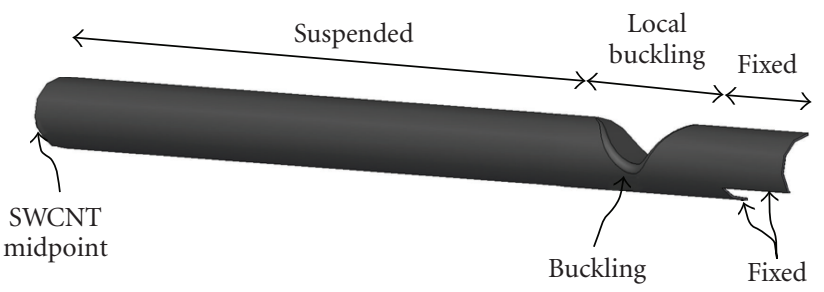

FIGURE 7: Schematic view of the FE model after the creation of the local buckling site. The three region of the SWCNT (fixed, hinge, and suspended areas) are shown.

in the lateral direction. As the slackness increases, this lateral component increases as well due to the larger slope of the SWCNT.

Next, the behavior of SWCNTs with different lengths and electrostatic gaps is shown in Figures 4 and 5, respectively, for $\Delta T=\Delta T_{\text {cr }}+250[K]$. Note that due to the geometric nonlinearity, the dependence between the force and the maximum deflection is nonlinear. We emphasize that the strains plotted in Figures 2(b), 4(b), and 5(b) can be as high as several millistrains. Such a significant strain was reported to have substantial effect on SWCNT electrical behavior $[5,7,9,18]$.

\section{Influence of the Loading on Local Buckling Sites}

The results shown in Section 3 demonstrate that resistive heating can cause the deflections to be in the order of hundreds of nanometers. The influence of such significant deflections was analyzed in this section. Here, we analyze how this thermal heating, and resulting large deflections, influence local buckling sites previously reported in suspended SWCNTs [28]. These buckling sites, shown schematically in Figure 6, may result either from intentional SWCNT loading or unintentional bending during the fabrication process. Buckling in the fabrication process originates mainly due to strong van der Waals forces, which are dominant surface forces in nanoscale structures (see $[13,17,28]$ for more information regarding surface forces and buckling in SWCNTs).

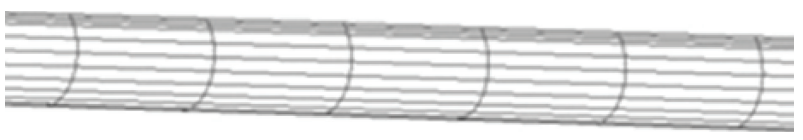

(a)

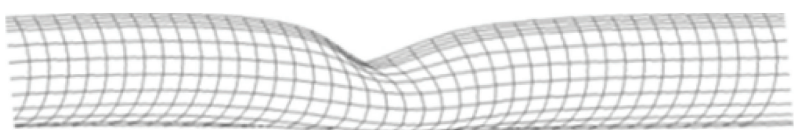

(b)

FIGURE 8: Finite element model built in ADINA software. (a) Site with no buckling. (b) Local buckling site.

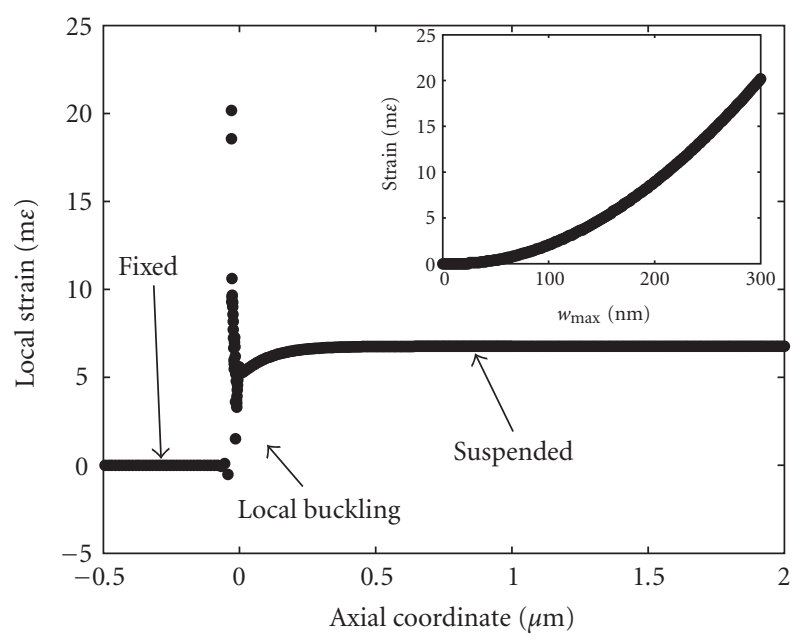

Figure 9: Strain versus the axial coordinate obtained from FE simulation for maximum SWCNT displacement of $300 \mathrm{~nm}$. Three regions are defined. The region where the SWCNT is fixed, the region which surrounds the buckling site, and the region where the SWCNT is suspended. Inset: local strain versus the maximum SWCNT displacement for the node of maximum strain.

To understand the effect of these buckling sites on SWCNT mechanics, an FE model was built in ADINA software. The SWCNT was meshed with large displacement shell elements. The symmetries of the problem were exploited to build compact model. Due to the transverse and longitudinal symmetries, only a quarter of a SWCNT was taken into the FE model. In addition, the boundary conditions include a $0.5 \mu \mathrm{m}$ long segment which is anchored (fixed) at its bottom. The other end, which represents the midpoint of the SWCNT, was free to deflect but was enforced to have a zero angle. A schematic description of the FE model is shown in Figure 7.

Initially, a SWCNT without buckling sites was built (Figure 8(a)). The simulation had two parts. In the first part, a displacement was enforced and remained constant in the area of the buckling site in order to create a structure similar to buckling sites observed in SWCNTs (Figure 8(b)). Next, displacements were applied to the midpoint of the SWCNT and were gradually increased until displacements of hundreds of nanometers were achieved. Strain presented in Figure 9 was calculated with respect to the state of 
zero displacement applied to the midpoint of the SWCNT, namely, with respect to the initial strain of the second part of the simulation.

The results of the FE simulation are shown in Figure 9. Three main regions are clearly identified. The first is the region that represents the strain in part of the SWCNT which is fixed (marked as "fixed" in Figures 7 and in 9), the second region is the part that surrounds the local buckling site (marked as "local buckling" in Figures 7 and in 9), and the last part is the suspended part of the SWCNT (marked as "suspended" in Figures 7 and in 9). We emphasize that the region that surrounds the local buckling site underwent a significant strain in the order of several tens of mili-strain. The relation between the local strain and the maximum SWCNT displacement is shown in the inset of Figure 9 for the node that demonstrated the maximum strain. Therefore, one can conclude that the local buckling sites can have an enhancing effect on strain generated in SWCNTs.

\section{Conclusions}

The mechanical behavior of SWCNT under resistive heating and electrostatic loading was analyzed. A SWCNT was modeled as a string, and a large displacement, nonlinear model was developed. The governing equations were solved, and model results show that with sufficient large resistive heating, SWCNT can buckle and thus may demonstrate significantly different mechanical behavior. The effect of the geometrical parameters (length and electrostatic gap) on the SWCNT behavior were also analyzed. Finally, the influence of local buckling sites was analyzed and was found to have an enhancing effect on the SWCNTs local strain. Therefore, the presented results provide an important insight into the unique mechanics of suspended SWCNTs.

\section{Acknowledgments}

The authors thank Eran Bahana for his help in schematics and Dr. Lior Kogut for helpful discussion.

\section{References}

[1] Y. A. Tarakanov and J. M. Kinaret, "A carbon nanotube field effect transistor with a suspended nanotube gate," Nano Letters, vol. 7, no. 8, pp. 2291-2294, 2007.

[2] A. K. Hüttel, G. A. Steele, B. Witkamp, M. Poot, L. P. Kouwenhoven, and H. S. J. Van Der Zant, "Carbon nanotubes as ultrahigh quality factor mechanical resonators," Nano Letters, vol. 9, no. 7, pp. 2547-2552, 2009.

[3] P. Hu, J. Zhang, LE. Li, Z. Wang, W. O'Neill, and P. Estrela, "Carbon nanostructure-based field-effect transistors for labelfree chemical/biological sensors," Sensors, vol. 10, no. 5, pp. 5133-5159, 2010.

[4] C. Stampfer, A. Jungen, R. Linderman, D. Obergfell, S. Roth, and C. Hierold, "Nano-electromechanical displacement sensing based on single-walled carbon nanotubes," Nano Letters, vol. 6, no. 7, pp. 1449-1453, 2006.

[5] R. C. Stampfer, T. Helbling, D. Obergfell et al., "Fabrication of single-walled carbon-nanotube-based pressure sensors," Nano Letters, vol. 6, no. 2, pp. 233-237, 2006.
[6] G. A. Karp, A. Ya'Akobovitz, M. David-Pur et al., "Integration of suspended carbon nanotubes into micro-fabricated devices," Journal of Micromechanics and Microengineering, vol. 19, no. 8, Article ID 085021, 2009.

[7] E. D. Minot, Y. Yaish, V. Sazonova, JI. Y. Park, M. Brink, and P. L. McEuen, "Tuning carbon nanotube band gaps with strain," Physical Review Letters, vol. 90, no. 15, pp. 1-4, 2003.

[8] H. Y. Chiu, P. Hung, H. W. C. Postma, and M. Bockrath, "Atomic-scale mass sensing using carbon nanotube resonators," Nano Letters, vol. 8, no. 12, pp. 4342-4346, 2008.

[9] N. K. Chang, C. C. Su, and S. H. Chang, "Fabrication of single-walled carbon nanotube flexible strain sensors with high sensitivity," Applied Physics Letters, vol. 92, no. 6, Article ID 063501, 2008.

[10] H. Maune and M. Bockrath, "Elastomeric carbon nanotube circuits for local strain sensing," Applied Physics Letters, vol. 89, no. 17, Article ID 173131, 2006.

[11] K. I. Tserpes and P. Papanikos, "Finite element modeling of single-walled carbon nanotubes," Composites Part B: Engineering, vol. 36, no. 5, pp. 468-477, 2005.

[12] D. A. Walters, L. M. Ericson, M. J. Casavant et al., "Elastic strain of freely suspended single-wall carbon nanotube ropes," Applied Physics Letters, vol. 74, no. 25, pp. 3803-3805, 1999.

[13] Q. Wang, K. M. Liew, X. Q. He, and Y. Xiang, "Local buckling of carbon nanotubes under bending," Applied Physics Letters, vol. 91, no. 9, Article ID 093128, 2007.

[14] J. P. Salvetat, G. A. D. Briggs, J. M. Bonard et al., "Elastic and shear moduli of single-walled carbon nanotube ropes," Physical Review Letters, vol. 82, no. 5, pp. 944-947, 1999.

[15] J. Cao, Q. Wang, and H. Dai, "Electromechanical properties of metallic, quasimetallic, and semiconducting carbon nanotubes under stretching," Physical Review Letters, vol. 90, no. 15, pp. 1-4, 2003.

[16] S. W. Lee, G. H. Jeong, and E. E. B. Campbell, "In situ raman measurements of suspended individual single-walled carbon nanotubes under strain," Nano Letters, vol. 7, no. 9, pp. 25902595, 2007.

[17] T. Hertel, R. E. Walkup, and P. Avouris, "Deformation of carbon nanotubes by surface van der Waals forces," Physical Review B, vol. 58, no. 20, pp. 13870-13873, 1998.

[18] G. Cao and X. I. Chen, "Buckling of single-walled carbon nanotubes upon bending: molecular dynamics simulations and finite element method," Physical Review B, vol. 73, no. 15, Article ID 155435, 2006.

[19] O. Liba, Y. Hanein, D. Kauzlaric, A. Greiner, and J. G. Korvink, "Investigationof the dynamic behavior of bridged nanotube resonators by dissipative particle dynamics simulation," International Journal for Multiscale Computational Engineering, vol. 6, no. 6, pp. 549-562, 2008.

[20] L. Y. Chan and B. Andrawes, "Finite element analysis of carbon nanotube/cement composite with degraded bond strength," Computational Materials Science, vol. 47, no. 4, pp. 994-1004, 2010.

[21] Y. Kuronuma, Y. Shindo, T. Takeda, and F. Narita, "Fracture behaviour of cracked carbon nanotube-based polymer composites: experiments and finite element simulations," Fatigue and Fracture of Engineering Materials and Structures, vol. 33, no. 2, pp. 87-93, 2010.

[22] V. V. Deshpande, S. Hsieh, A. W. Bushmaker, M. Bockrath, and S. B. Cronin, "Spatially resolved temperature measurements of electrically heated carbon nanotubes," Physical Review Letters, vol. 102, no. 10, Article ID 105501, 2009. 
[23] A. Ya'akobovitz, G. Karp, M. David-Pur, S. Krylov, and Y. Hanein, "Carbon nanotube self-assembeled high frequency resonator," in Proceedings of the IEEE 23rd International Conference on Micro Electro Mechanical Systems (MEMS '10), Hong-Kong, China, 2010.

[24] A. Ya'akobovitz, G. Karp, Y. Hanein, and S. Krylov, "Electromechanical behavior of suspended taut single-walled carbon nanotubes," in Proceedings of the International IEEE Conference on Microwaves, Communications, Antennasand Electronic Systems (COMCAS '09), Tel-Aviv, Israel, 2009.

[25] S. Senturia, Microsystem Design, Kluwer Academic, Boston, Mass, USA, 2001.

[26] E. Weber, Electromagnetic Theory : Static Fields and Their Mapping, Dover, New York, NY, USA, 1965.

[27] H. Jiang, B. Liu, Y. Huang, and K. C. Hwang, "Thermal expansion of single wall carbon nanotubes," Journal of Engineering Materials and Technology, Transactions of the ASME, vol. 126, no. 3, pp. 265-270, 2004.

[28] Z. R. Abrams, Y. Lereah, and Y. Hanein, "Transmission electron microscope imaging of single-walled carbon nanotube interactions and mechanics on nitride grids," Nanotechnology, vol. 17, no. 18, pp. 4706-4712, 2006. 

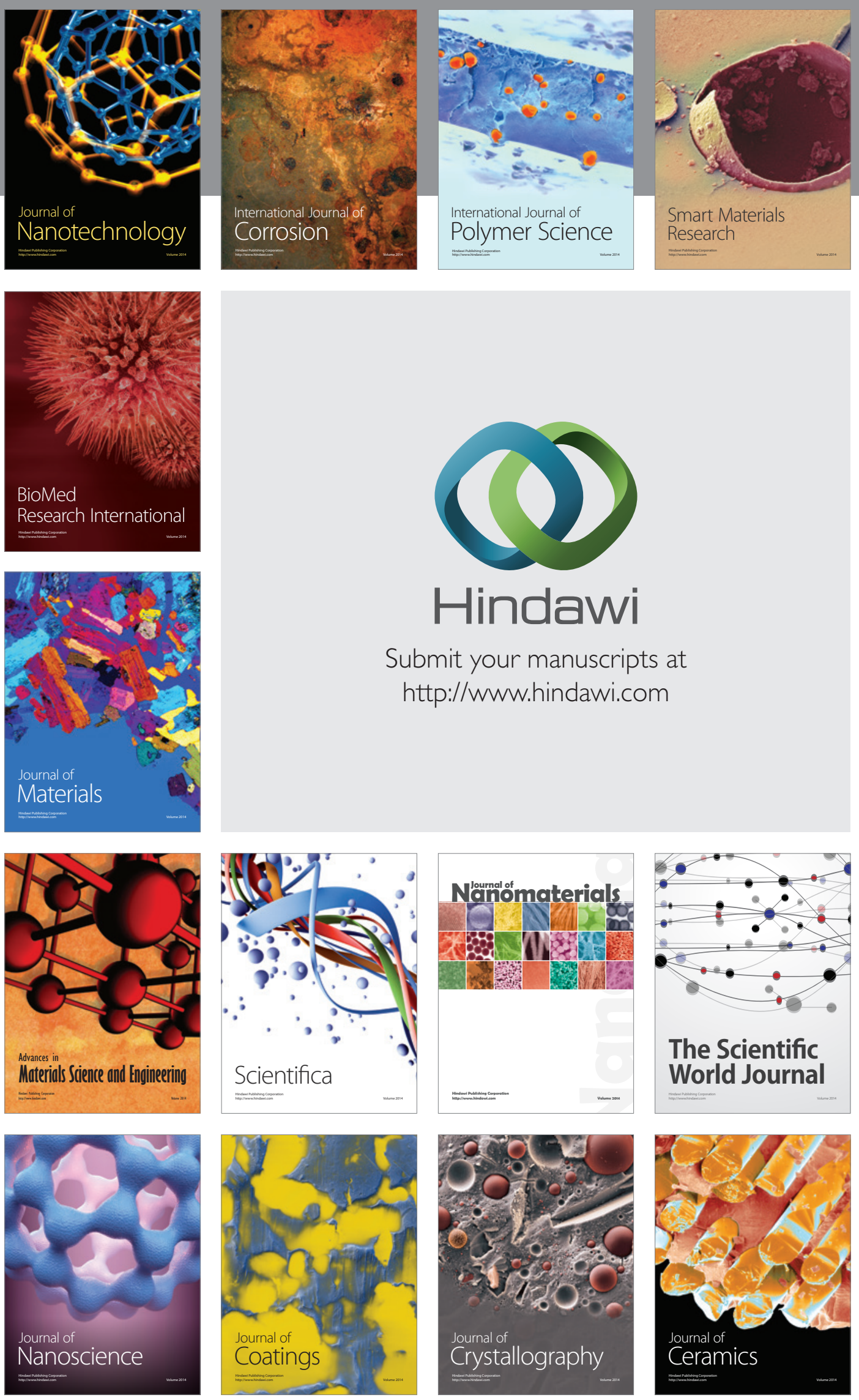

The Scientific World Journal

Submit your manuscripts at

http://www.hindawi.com

\section{World Journal}

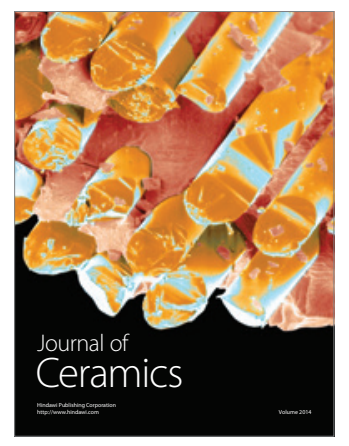

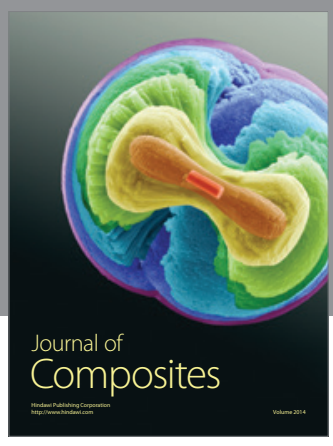
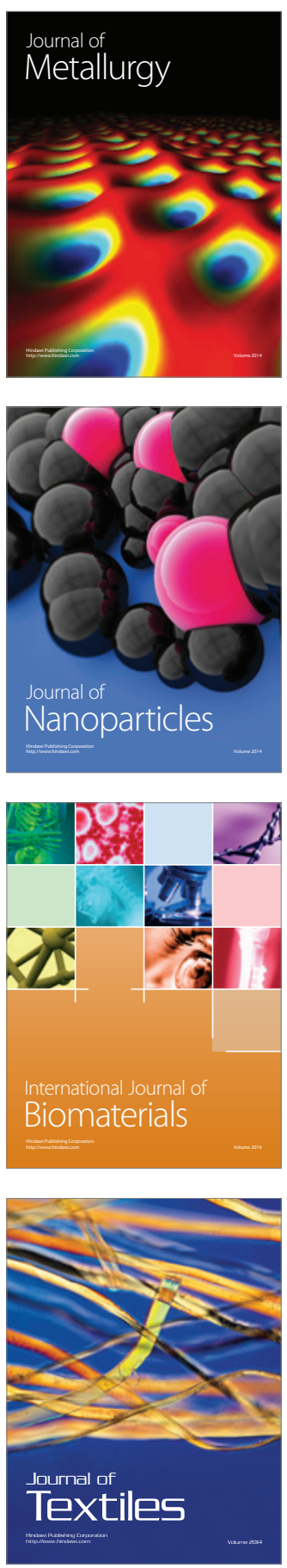